\title{
Morphing Structure for a Rudder
}

\author{
Miguel Á. Castillo-Acero \\ VP Technology Development Aernnova
}




\section{AERnnOVa}

\section{Index}

- Motivation

- Methodology

- Results and Discussion

- Conclusions 


\section{Motivation}

- Commercial aeronautics chain is under pressure to optimize our operations: Direct Operating Costs, Fuel efficiency, CO2 emissions, etc.

- New Technology is one of the pillars to cope with this challenge.

- Smart materials and actuators enable new airfoils controlled deformation.

- In previous work, it has been quantified the aerodynamic improvements that can be achieved with an unconventional morphing rudder.

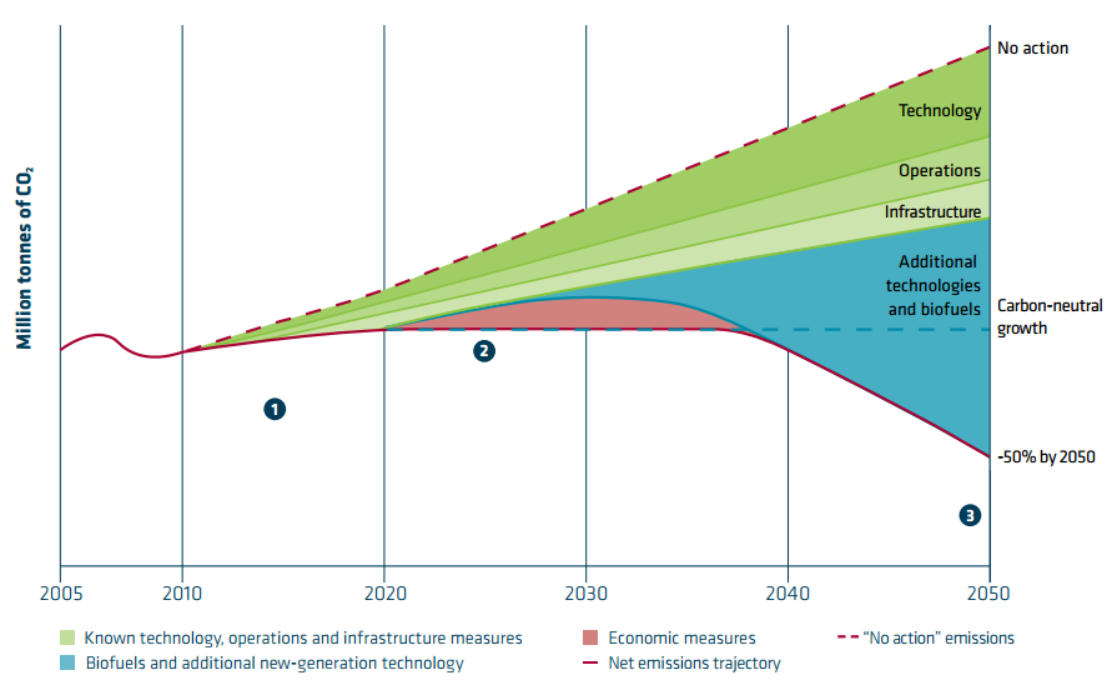

Air Traffic Action Group, "A sustainable flightpath to reduce emissions. UNFCCC Climate Talks, Doha,” Nov. 2012.

- New materials for 3D printing enable novel morphing structures structurally compliant.

This paper demonstrates the structural feasibility of a novel morphing rudder based on morphing skins 3D printable. 


\section{Methodology (I)}

- The methodology is based on the preliminary sizing of the new non-conventional rudder.

- The sizing load case is engine failure during takeoff for an objective commercial plane.

- The differential pressure distribution in the vertical tail plane section at $\mathrm{I} / 3$ height VTP span comes from previous studies.

- These aerodynamic loads are translated into:

" I(y): lifting forces per unit, span, length, i.e. lateral " $y$ " forces

$$
l(y)=\frac{1}{2} \rho v^{2} c \sum c_{l}\left(x_{i} / c\right) \Delta\left(x_{i} / c\right)
$$

b $m(z)$ : "z" torsion moments per unit, span, length

$$
m(z)=\frac{1}{2} \rho v^{2} c^{2} \sum c_{l}\left(x_{i} / c\right)\left(x_{i} / c-x_{l} / c\right) \Delta\left(x_{i} / c\right)
$$

- These loads have been discretized along the rudder span.

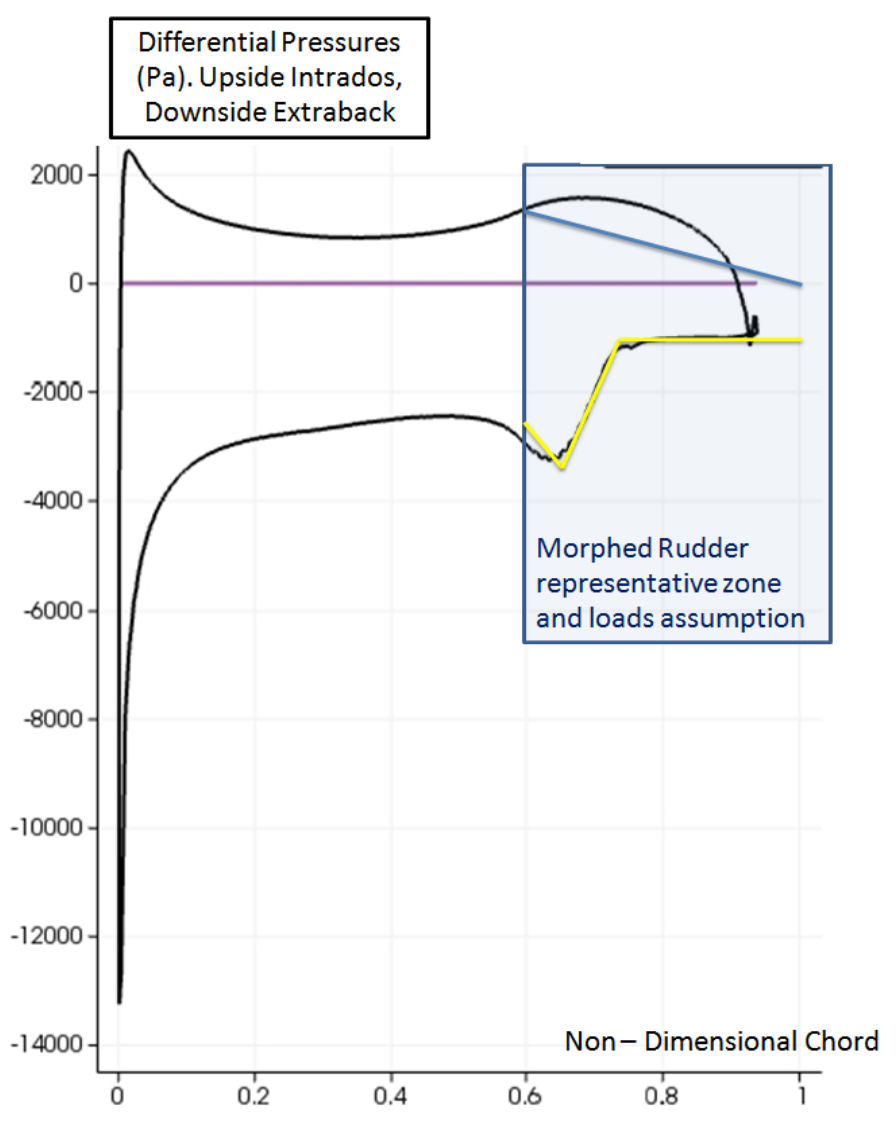

Classical bending- torsion box strength analysis approach, to obtain the critical internal loads in the morphed rudder structure and the maximum reactions at the fittings. 


\section{Methodology (II)}

- The kinematic of the morphing rudder is based on articulated ribs.

- They are composed by riblets that rotate when actuated.

- An optimization study of the number and configuration of these riblets is performed.

- The configuration with more riblets assures better adaptation to the target curve shape and therefore, better aerodynamic efficiency.

- However, this approach increases the weight.

- The optimum is achieved balancing these two trends.

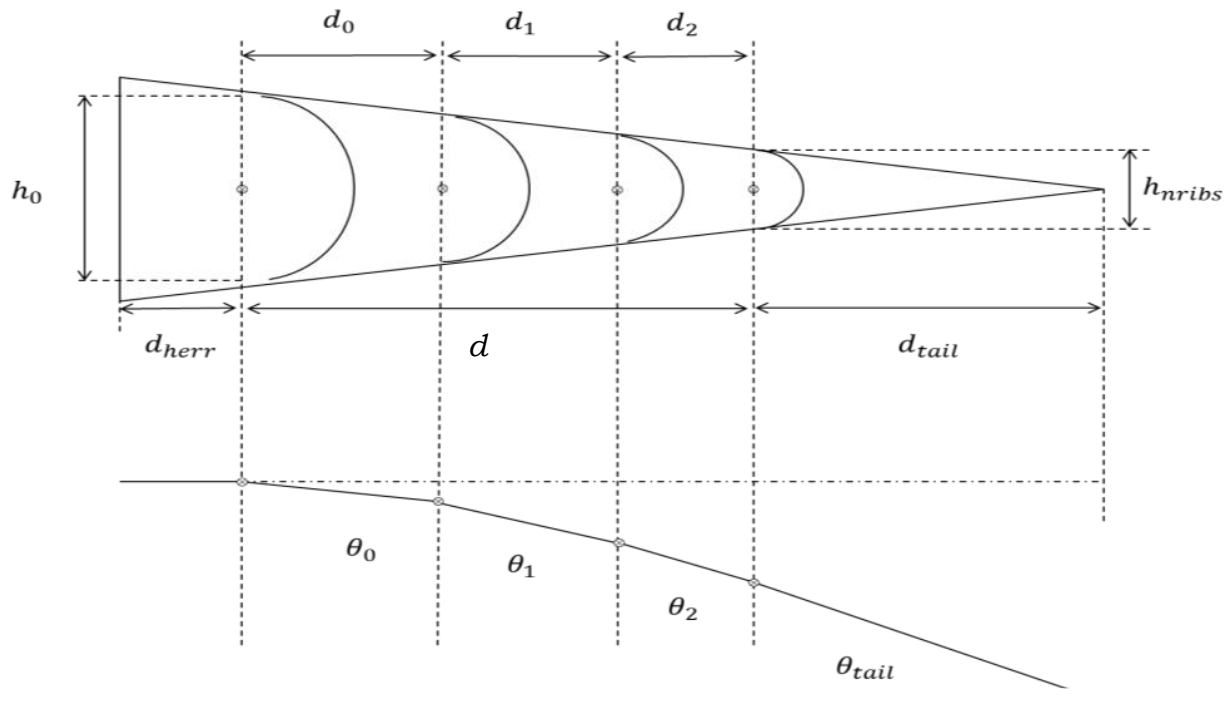

Parameters for this analysis: $d_{0}, \theta_{0}, k_{1}, k_{2}, n_{\text {ribs }}, d_{\text {herr }}, d_{\text {tail }}$

Geometrical imposed laws for rotations on the articulated ribs and riblets geometry:

Constraints:

$$
\begin{aligned}
\theta_{i+1}=k_{1} \theta_{i} & \rightarrow \theta_{i}=\left(k_{1}\right)^{i} \theta_{0} \\
d_{i+1}=k_{2} d_{i} & \rightarrow d_{i}=\left(k_{2}\right)^{i} d_{0}
\end{aligned}
$$

$d_{A C}=d_{\text {herr }}+d_{\text {tail }}+\sum_{0}^{n_{\text {ribs }}} d_{i} \rightarrow d_{A C}=d_{\text {herr }}+d_{\text {tail }}+d_{0} \frac{k_{2}^{n_{\text {ribs }-1}}}{k_{2}-1}$

$d_{A C} \sin 30=d_{\text {herr }} \sin 0+d_{\text {tail }} \sin \theta_{\text {tail }}+\sum_{0}^{n_{\text {ribs }}} d_{i} \sin \theta_{i} \rightarrow \frac{d_{A C}}{2}=d_{\text {tail }} \sin \theta_{n_{\text {ribs }}+1}+\sum_{0}^{n_{\text {ribs }}}\left(k_{2}\right)^{i} d_{0} \sin \left(\left(k_{1}\right)^{i} \theta_{0}\right)$ $\frac{d_{0}}{h_{0}}=\frac{d_{0} k_{2}}{h_{1}}=\frac{d_{0}\left(k_{2}\right)^{2}}{h_{2}} \cdots=\frac{d_{0}\left(k_{2}\right)^{n_{\text {ribs }}}}{h_{n_{\text {ribs }}}} \rightarrow \frac{h_{n_{\text {ribs }}}}{h_{0}}=\frac{d_{\text {tail }} \sin \alpha}{h-d_{\text {herr }} \sin \alpha}=\left(k_{2}\right)^{n_{\text {ribs }}}$ 


\section{AERnnOVa}

\section{Methodology (III)}

- Skins are cell based skeletons that span-wise present higher stiffness than chord-wise.

- 3D printable material Visijet M3 X

- Two different geometric configurations to maximize elastic moduli $\mathrm{E}_{1}, \mathrm{E}_{2}, \mathrm{E}_{3}$ \& minimum weight.

- The geometric parameters involved in the skins strength analysis are $t, t_{s}$ stringer thickness, $b$ distance between stringers and $d$.

\begin{tabular}{llc} 
Properties & Condition & $\begin{array}{c}\text { VisiJet M3 } \\
\mathbf{X}\end{array}$ \\
\hline Density @ $80^{\circ} \mathrm{C}$ (liquid) & ASTM D4164 & $1.04 \mathrm{~g} / \mathrm{cm}^{3}$ \\
\hline Tensile Strength & ASTM D638 & $49 \mathrm{MPa}$ \\
\hline Tensile Modulus & ASTM D638 & $2168 \mathrm{MPa}$ \\
\hline Elongation at Break & ASTM D638 & $8.3 \%$ \\
\hline Flexural Strength & ASTM D638 & $65 \mathrm{MPa}$ \\
\hline $\begin{array}{l}\text { Heat Distortion Tem- } \\
\text { perature @ 0.45MPa }\end{array}$ & ASTM D648 & $88^{\circ} \mathrm{C}$ \\
\hline
\end{tabular}
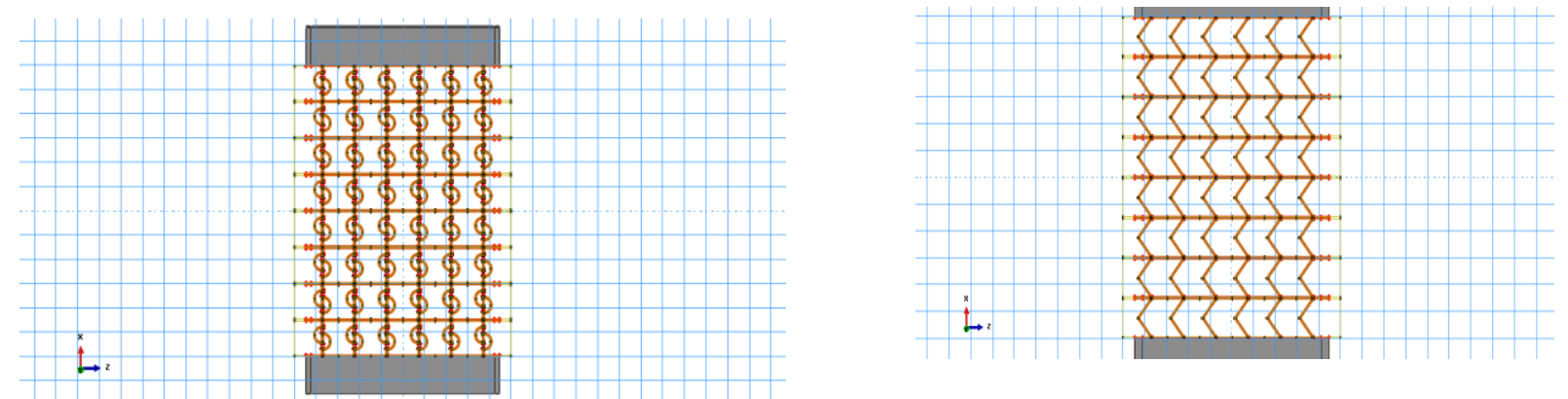

Visijet $M 3 \quad X \quad 3 D$ printable material

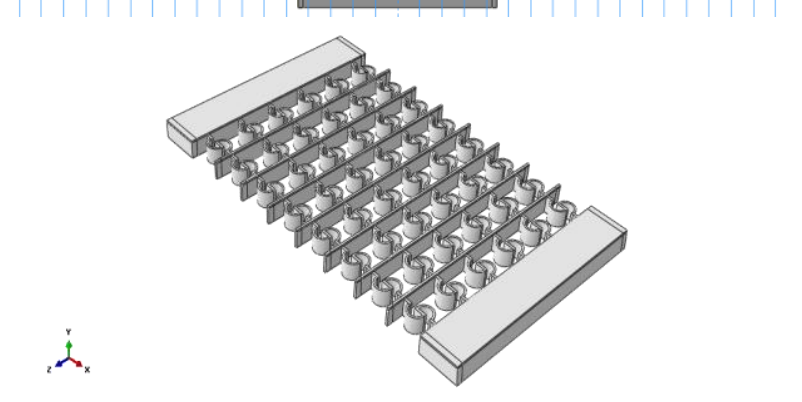

Novel Double C configuration

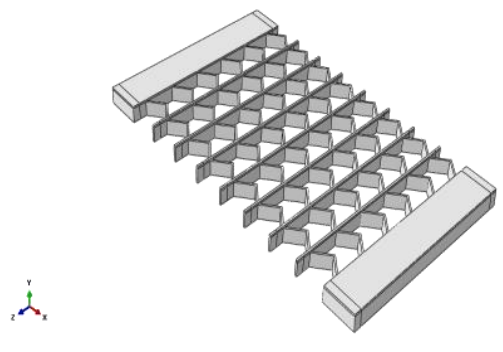

Reference V Straight configuration 


\section{Methodology (IV)}

- Skins span-wise critical condition for sizing is compression internal loading and so, the critical failure mode is buckling.

- Equivalent material properties for single ply skin orthotropic material analysis.

- Farrar theory applied: optimum skin is the one in which the compression stresses at the beginning of the initial local buckling match the corresponding Euler buckling as a column.

- The Farrar theory also defines a reference stress, and a mean stress, because the reinforced skin stress distribution is not uniform.

- Farrar factor, $F$, is defined as the ratio between the mean and the reference stress, concluding that the higher the value of $F$, the most optimum the structure results.

- The Farrar equations have been Matlab programmed.

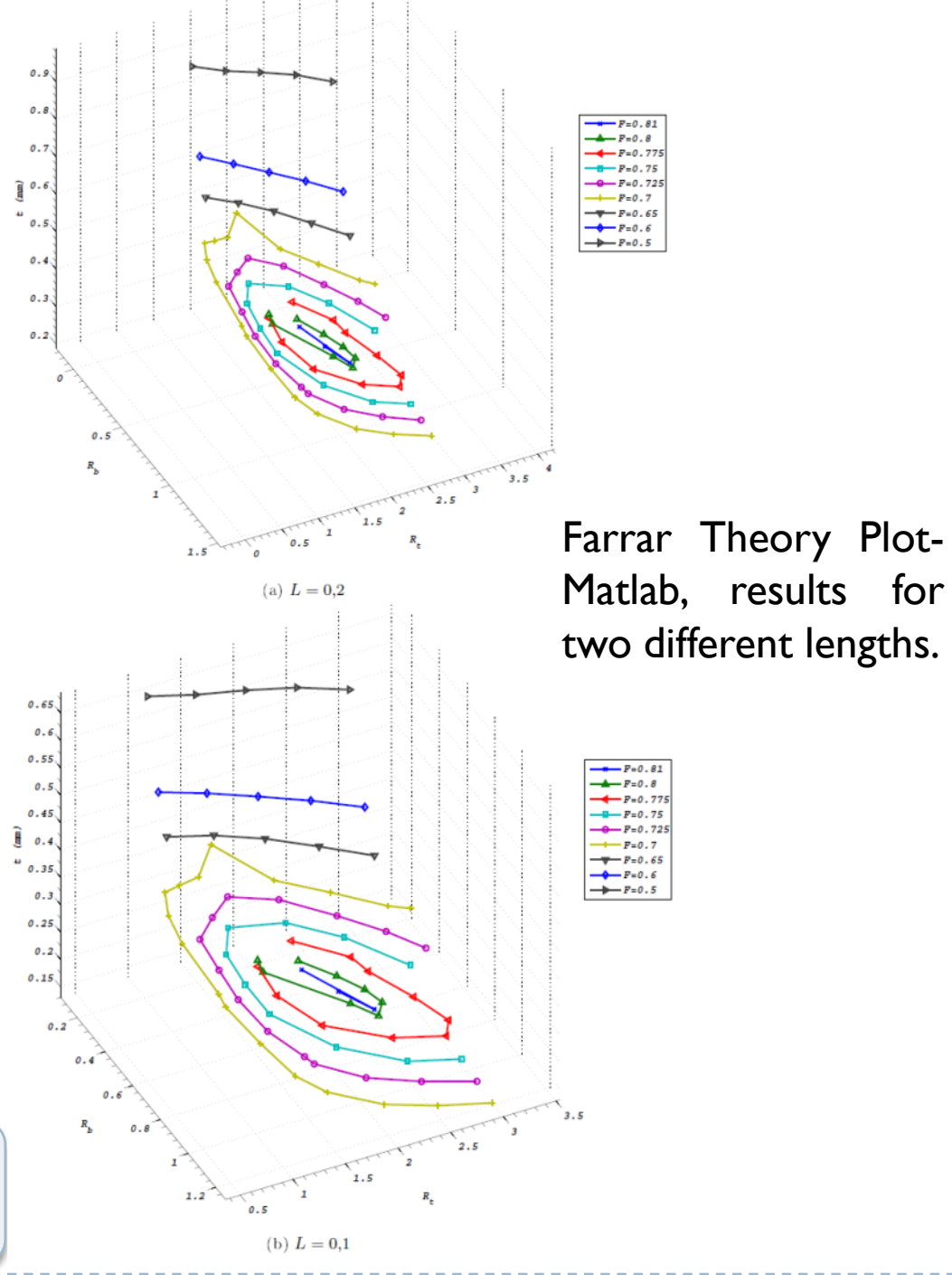

(b) $L=0,1$ 


\section{Methodology (IV)}

- The skin chord-wise can deform, as a skeleton, up to certain level to accommodate the morphing.

- To characterize the strength, the maximum stress at break under tension is considered, and the tensile stiffness.

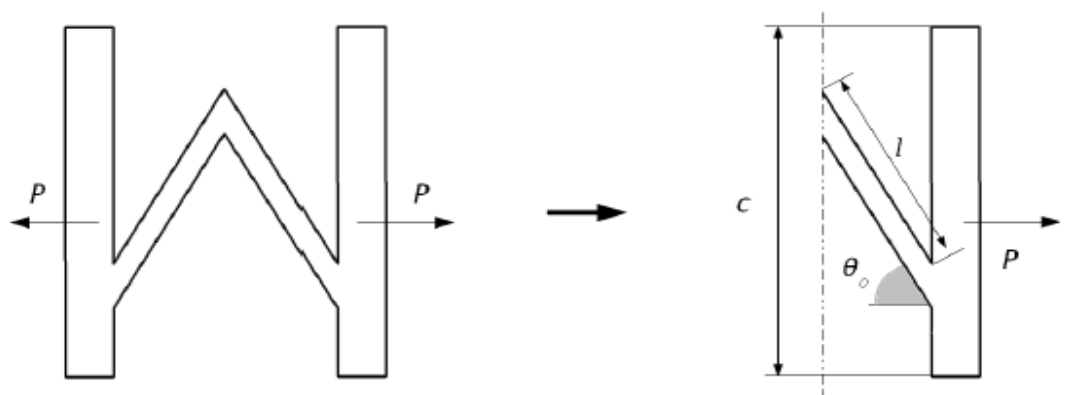

- To calculate the elastic module of the new configuration in Double-C, a number of assumptions have been considered.

- The theory of Euler - Bernoulli should be applicable. This means that the deformations are sufficiently small so that the sections originally perpendicular to the line of deformation, remain perpendicular after deformation.

- Using symmetry conditions the analysis is simplified just imposing the appropriate boundary conditions in each case. 


\section{AERnnOVa}

\section{Results and Discussion (I)}

- Articulated Ribs. Optimization set for 3 riblets to avoid mechanism complexities.

\begin{tabular}{ccccccccccc}
$n_{\text {ribs }}$ & $k_{2}$ & $\theta_{\text {tail }}$ & $\Delta \theta$ & $\theta_{0}$ & $h_{n_{\text {ribs }}}(m)$ & $h_{0}(m)$ & $d_{A C}(m)$ & $d_{0}(m)$ & $d_{\text {herr }}(m)$ & $d_{\text {tail }}(m)$ \\
\hline 3 & 0,61 & 0,35 & 0,15 & 0,3 & 0,1 & 0,27 & 0,8 & 0,3 & 0,13 & 0,2 \\
\hline
\end{tabular}

- Skins. Equivalent single layer panel results for minimum weight.

\begin{tabular}{cccccccc}
$t_{s}(\mathrm{~mm})$ & $d(\mathrm{~mm})$ & $q_{c r}(\mathrm{~N} / \mathrm{m})$ & $\sigma_{V M}(\mathrm{MPa})$ & $F$ & $R_{b}$ & $R_{t}$ & $t / b$ \\
5.7 & 55.4 & 30500 & 27.0 & 0.75 & 0.6 & 1.25 & $4.6 / 92.4$ \\
\hline
\end{tabular}

Chord wise Springs parameters and Young Moduli,V Straight up, Double C bottom.

\begin{tabular}{cccccccc}
$n_{c}$ & $\theta_{0}(\stackrel{\mathrm{o}}{)})$ & $l(\mathrm{~mm})$ & $c(\mathrm{~mm})$ & $K(\mathrm{~N} / \mathrm{m})$ & $E_{1}(\mathrm{MPa})$ & $E_{2}(\mathrm{GPa})$ & $E_{3}(\mathrm{GPa})$ \\
\hline 6 & 59,5 & 10 & 106.5 & 55,4 & 0,175 & 0,231 & 0,445 \\
\hline$n_{c}$ & $\theta_{0}(\stackrel{\mathrm{o}}{)})$ & $l(\mathrm{~mm})$ & $c(\mathrm{~m})$ & $K(\mathrm{~N} / \mathrm{m})$ & $E_{1}(\mathrm{MPa})$ & $E_{2}(\mathrm{GPa})$ & $E_{3}(G P a)$ \\
\hline 6 & $\mathrm{n} / \mathrm{a}$ & 17 & 106.5 & 124,7 & 1,842 & 0,231 & 0,388 \\
\hline
\end{tabular}




\section{AERnnOVa}

\section{Results and Discussion (II)}

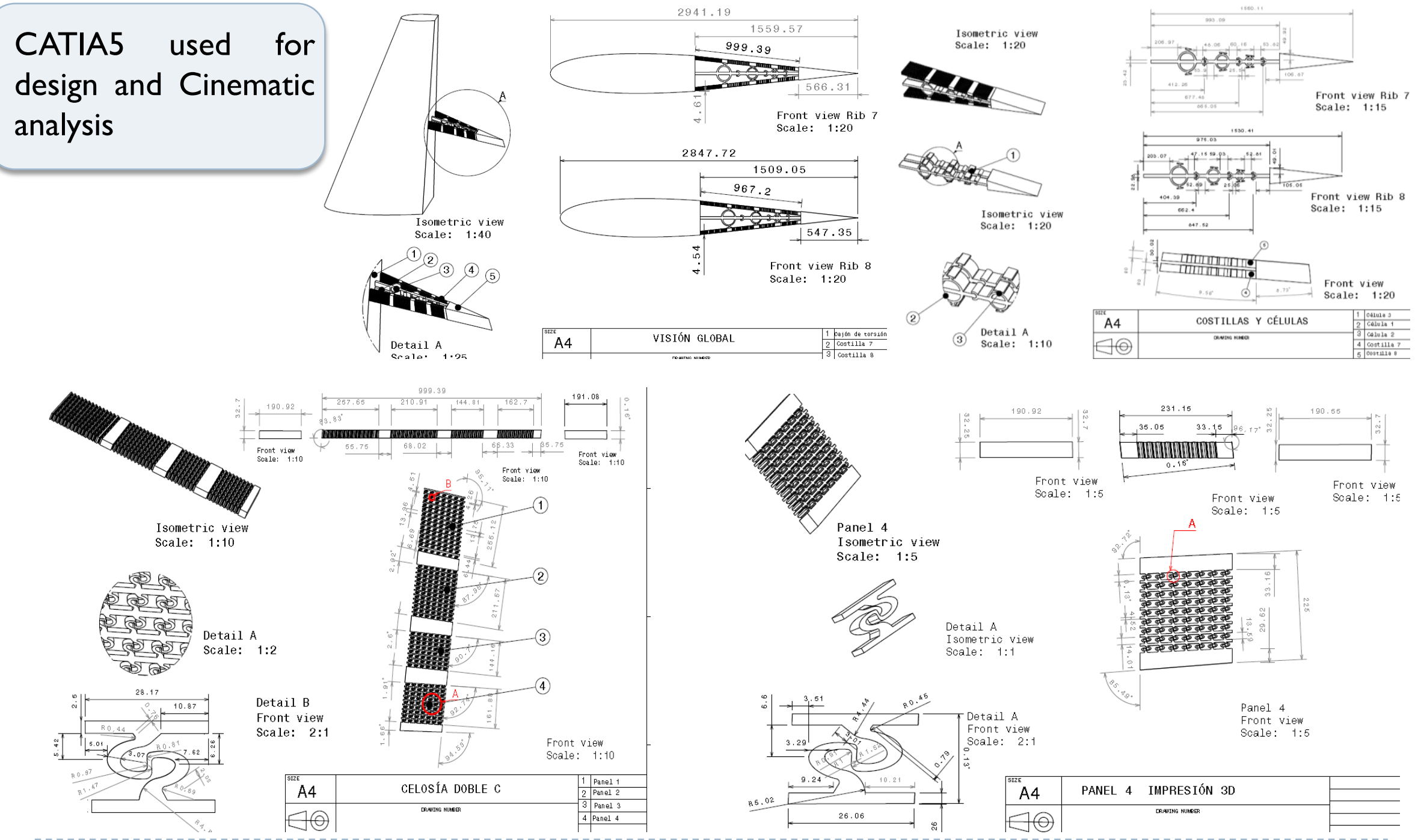




\section{Results and Discussion (III)}

- Numerical models based on the finite element method code ABAQUS.

- Account the geometrical parameters obtained, as well as the boundary conditions of the problem.

- Abaqus FEM models with rough meshing, but enough detail to test the two types of skeletons, excluding the exterior elastomeric skin, have been constructed.

- Then the mesh is refined to test the full skin for the two configurations.

- The model has been made for three consecutive ribs, so that the simulation results in the central rib is as representative as possible of the typical loads that stand most rudder ribs.

- The geometry of the second rib is considered because, as shown before, presents the highest internal loads.
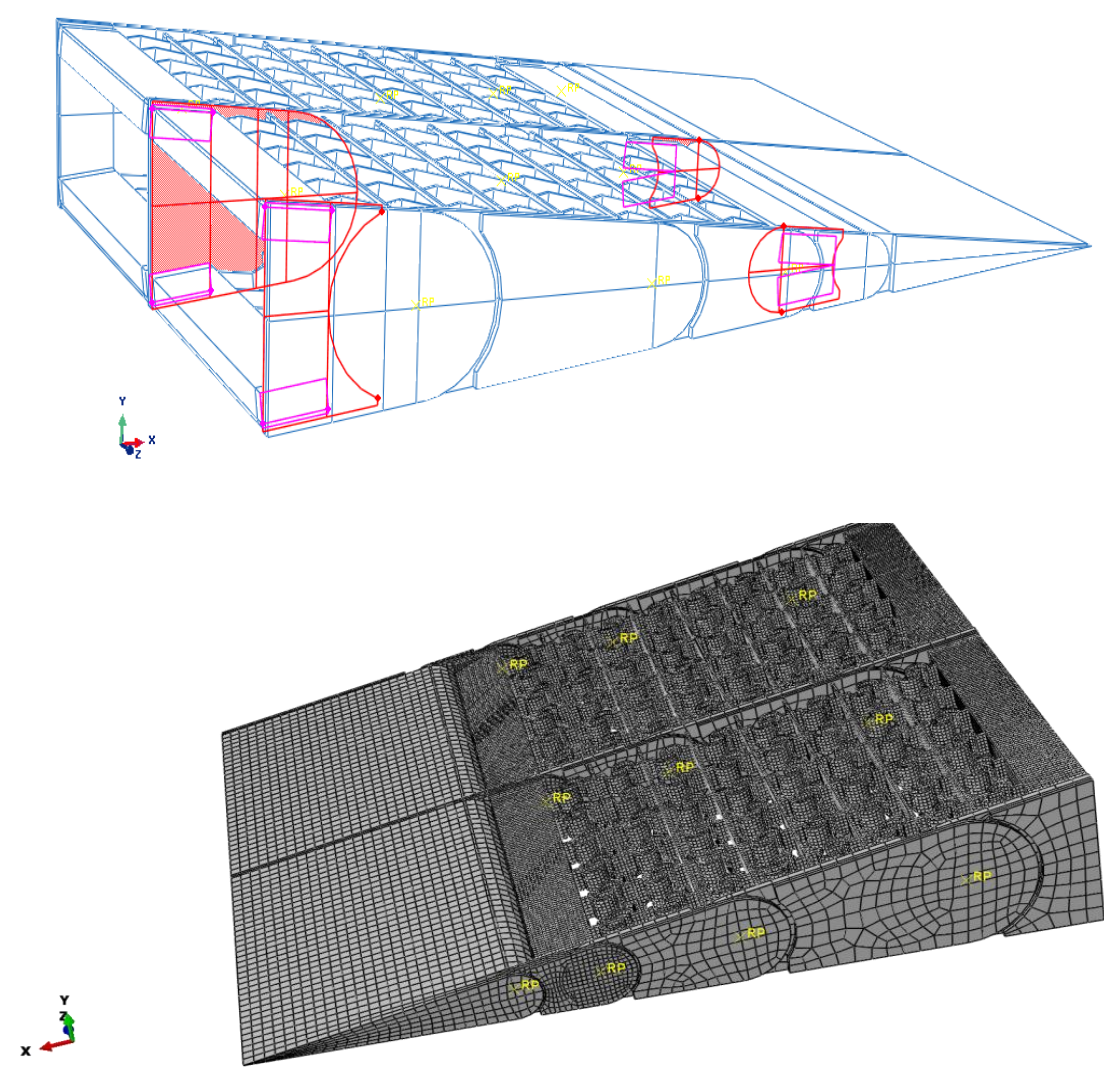

Abaqus 2.5D FEM Model general view. 


\section{AERnnOVa}

\section{Results and Discussion (III)}

"V-Straight" FEM Von Misses Stresses

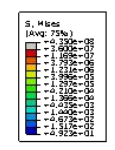

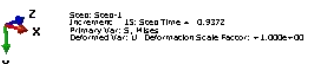

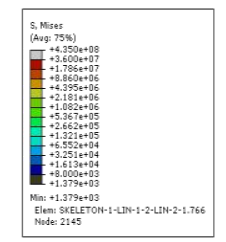

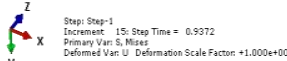

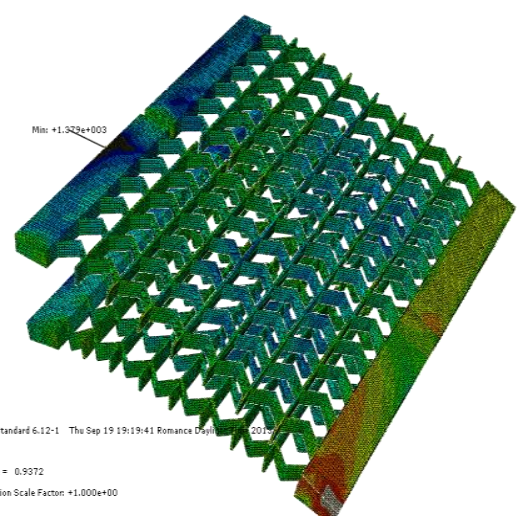

“Double-C” FEMVon Misses Stresses
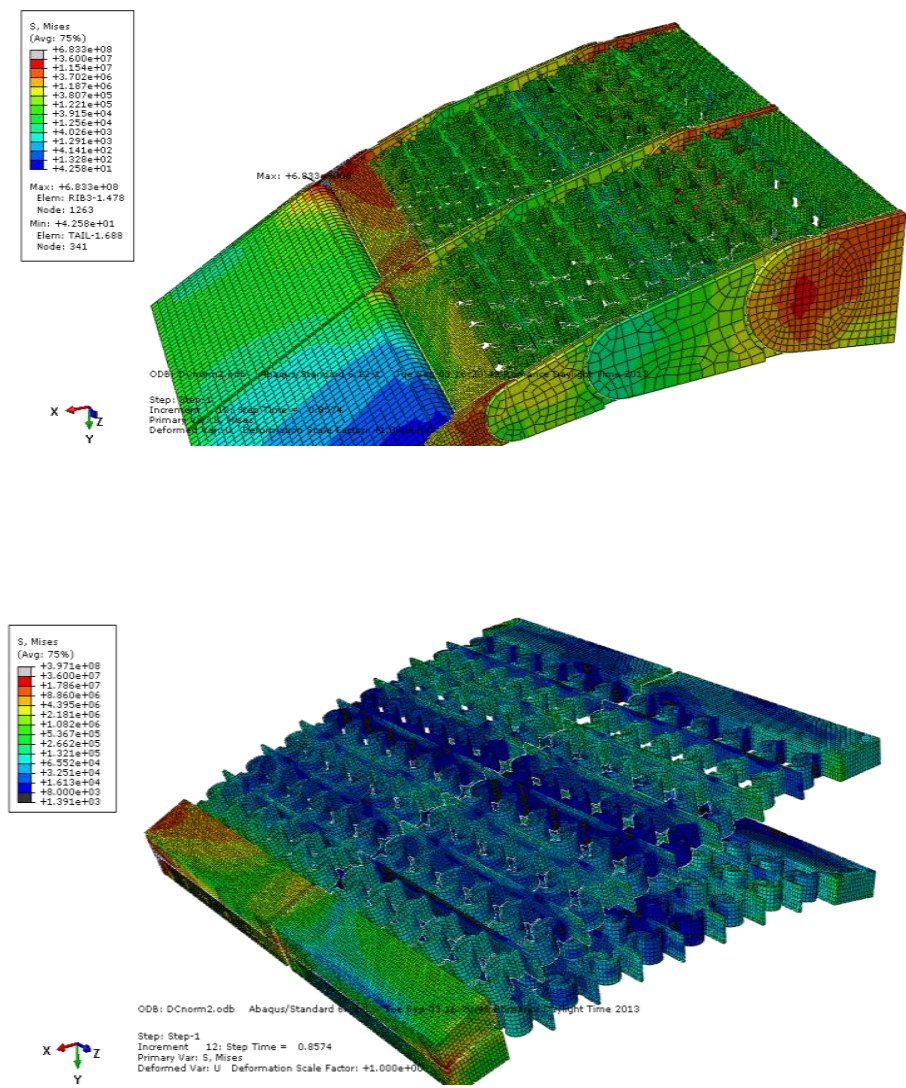

Double-C Skin FEM results on $90 \%$ reduction of maximum Von Misses stress. 


\section{Results and Discussion (IV)}

"V-Straight" FEM Strains

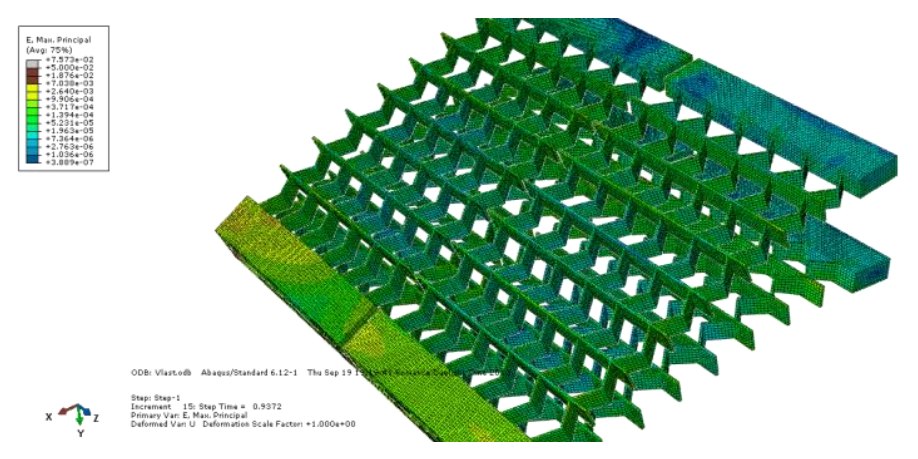

- It is relevant to note that stresses are much less concentrated in Double $\mathrm{C}$ configuration than in the V-Straight.

- This is critical from the point of view of the durability of the structure and compensates for its higher stiffness.

- These concentrations need to be further studied from fatigue point of view.

- Double C springs rotations are observed.
“Double-C” FEM Strains
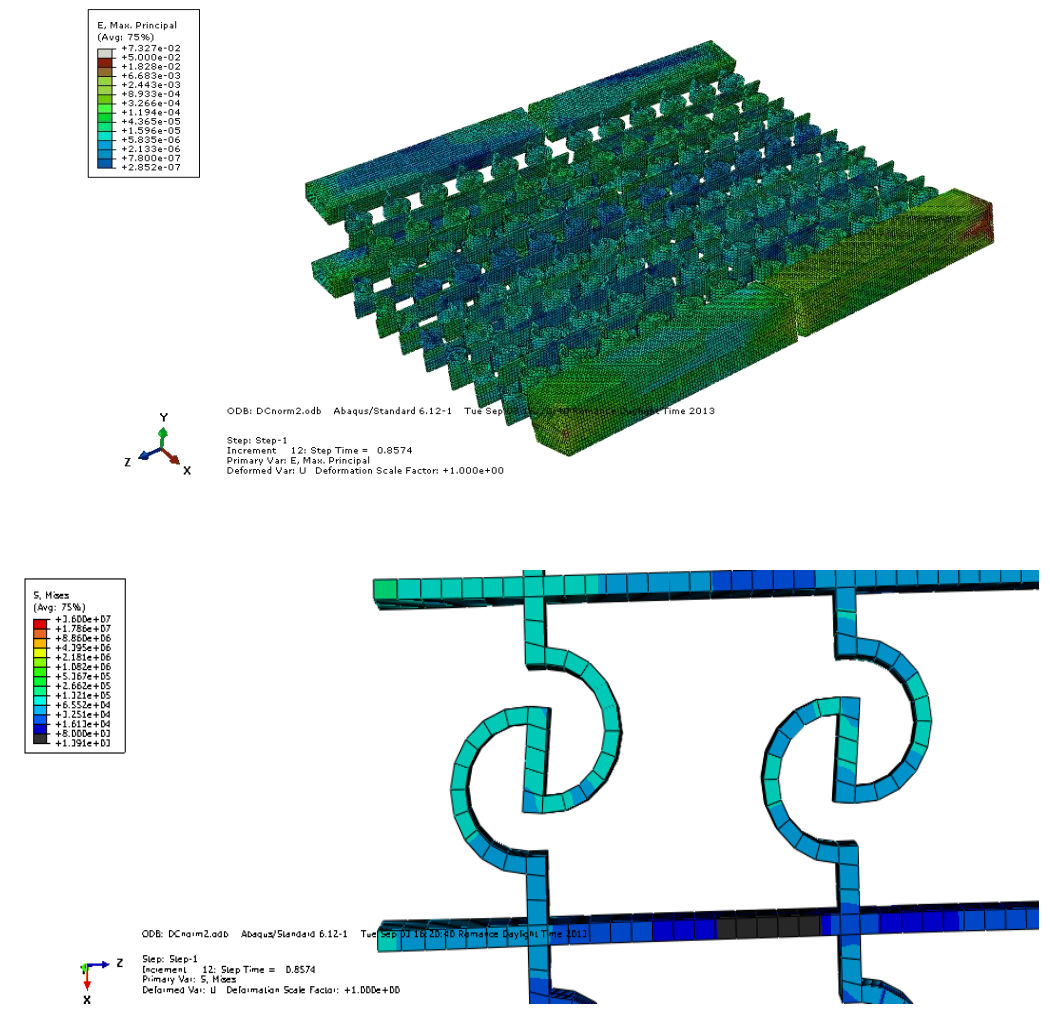

“Double-C” FEM spring rotation detail 


\section{AERnnOVa}

\section{Results and Discussion (V)}

- A 3D printing a "Double-C" representative panel in the selected material Visijet M 3X has been produced.

- The 3D printer is Projet $₫ 7000$ MP.

- It is based on stereo-lithography technology.

- The amount of material used is 210 grams.

- The time consumed on this printing has been 7 hours.
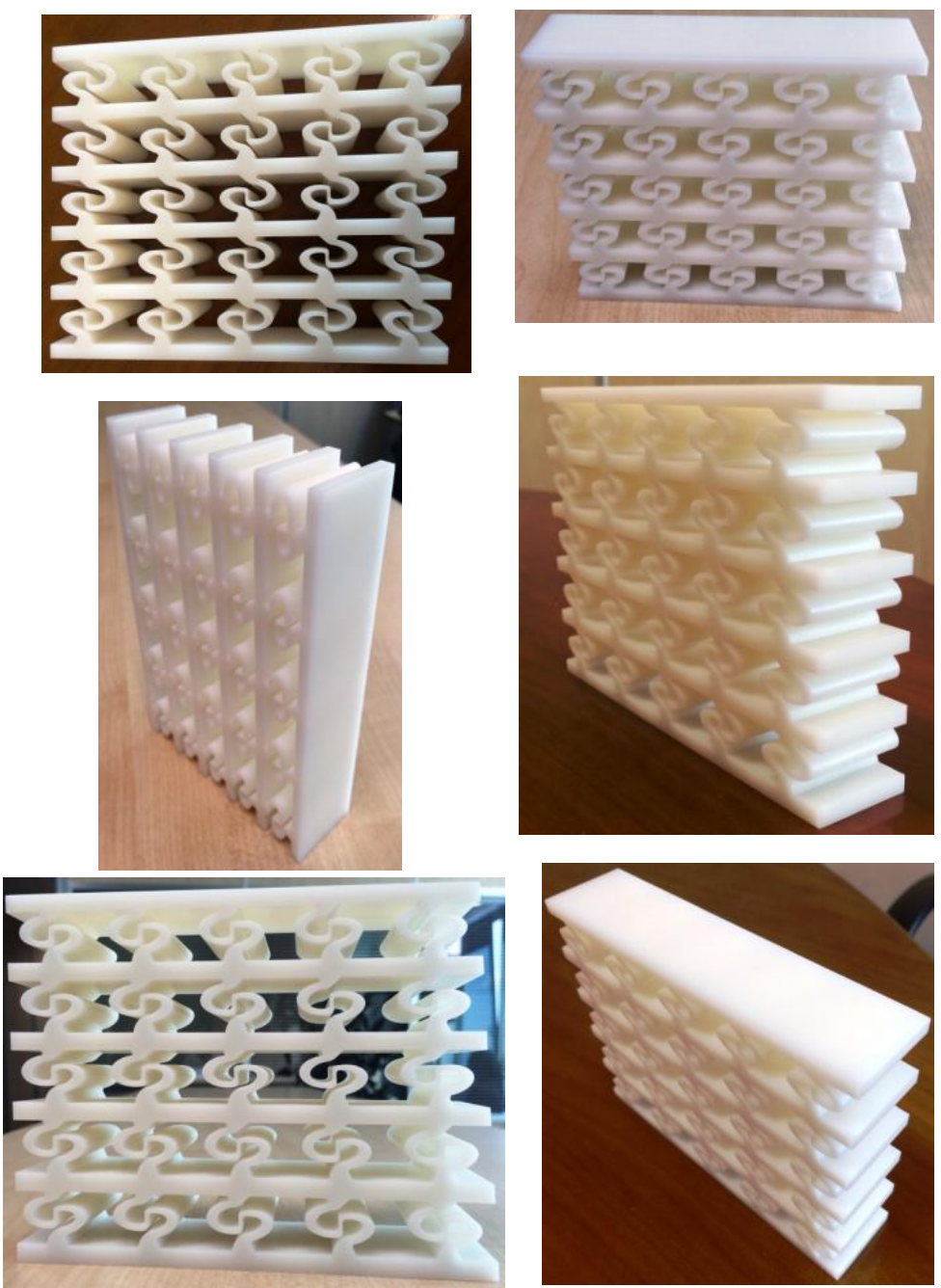


\section{Conclusions}

$\checkmark$ Equations that relate morphing skin 3D printable skeleton mechanical properties, with actual Zero Poison geometry and material, have been obtained.

$\checkmark$ The morphing skin has been integrated into CATIA 5 models, cinematically checked and strength analyzed with ABAQUS

$\checkmark$ After 3D printing a representative specimen, it is concluded that the design is feasible.

$\checkmark$ The selection of materials is critical to achieve a structurally compliant solution.

$\checkmark$ The resultant skin skeleton elastic modules have to be high enough to withstand the internal loads but not so big that the required actuators forces are beyond current available commercial servo-motor, during the deformation process.

$\checkmark$ Further studies are required to search for more adequate 3D printable materials

$\checkmark$ The "Double-C" skin configuration improve the "V-Straight" mechanical properties.

$\checkmark$ The stresses, strains, forces and moments obtained from ABAQUS results compared to the analytical models show some discrepancies that need further studies.

$\checkmark$ The characterization of fatigue life and the effect of relevant environment are pending, as well as the study on the highly deformable material and its integration to the skins for impermeability purposes.

$\checkmark$ The current commercially available 3-D printing machines are a limit to critical dimensions, in particular the size of the panels, being the maximum size currently available around 0.5 meters 


\section{AERnnOVa}

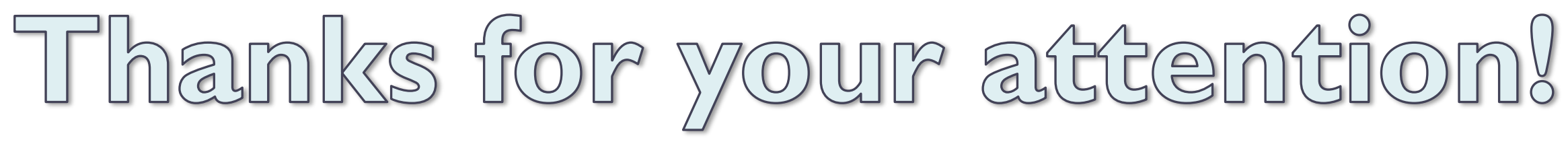
O)uesinons?

\section{Morphing Structure for a Rudder}

Miguel Á. Castillo-Acero

VP Technology Development Aernnova 